\title{
Far red/near infrared light-induced protection against cardiac ischemia and reperfusion injury remains intact under diabetic conditions and is independent of nitric oxide synthase
}

\author{
Agnes Keszler ${ }^{1}$, Garth Brandal ${ }^{1}$, Shelley Baumgardt ${ }^{1}$, Zhi-Dong Ge ${ }^{1}$, Phillip F. Pratt ${ }^{1}$, \\ Matthias L. Riess ${ }^{1,2,3}$ and Martin Bienengraeber ${ }^{1,4}$ *
}

${ }^{1}$ Department of Anesthesiology, Medical College of Wisconsin, Milwaukee, WI, USA

2 Department of Anesthesiology, Clement J. Zablocki VA Medical Center, Milwaukee, WI, USA

${ }^{3}$ Department of Physiology, Medical College of Wisconsin, Milwaukee, WI, USA

${ }^{4}$ Department of Pharmacology and Toxicology, Medical College of Wisconsin, Milwaukee, WI, USA

\section{Edited by:}

Miguel A. Aon, Johns Hopkins

University School of Medicine, USA

Reviewed by:

Fadi G. Akar, Mount Sinai School of

Medicine, USA

Derek John Hausenloy, University

College London, UK

Sruti Shiva, University of Pittsburgh, USA

*Correspondence:

Martin Bienengraeber, Medical College of Wisconsin,

Anesthesiology Research, 8701

Watertown Plank Road, Milwaukee,

WI 53226, USA

e-mail: mbieneng@mcw.edu
Far red/near-infrared light (NIR) promotes a wide range of biological effects including tissue protection but whether and how NIR is capable of acutely protecting myocardium against ischemia and reperfusion injury in vivo is not fully elucidated. Our previous work indicates that NIR exposure immediately before and during early reperfusion protects the myocardium against infarction through mechanisms that are nitric oxide (NO)-dependent. Here we tested the hypothesis that NIR elicits protection in a diabetic mouse model where other cardioprotective interventions such as pre- and postconditioning fail, and that the protection is independent of nitric oxide synthase (NOS). NIR reduced infarct size dose dependently. Importantly, NIR-induced protection was preserved in a diabetic mouse model (db/db) and during acute hyperglycemia, as well as in endothelial NOS ${ }^{-1-}$ mice and in wild type mice treated with NOS inhibitor L-NAME. In in vitro experiments NIR light liberates $\mathrm{NO}$ from nitrosyl hemoglobin ( $\mathrm{HbNO}$ ) and nitrosyl myoglobin (MbNO) in a wavelength- $(660-830 \mathrm{~nm})$ and dose-dependent manner. Irradiation at $660 \mathrm{~nm}$ yields the highest release of NO, while at longer wavelengths a dramatic decrease of NO release can be observed. Similar wavelength dependence was observed for the protection of mice against cardiac ischemia and reperfusion injury in vivo. NIR-induced NO release from deoxymyoglobin in the presence of nitrite mildly inhibits respiration of isolated mitochondria after hypoxia. In summary, NIR applied during reperfusion protects the myocardium against infarction in an NO-dependent, but NOS-independent mechanisms, whereby mitochondria may be a target of NO released by NIR, leading to reduced reactive oxygen species generation during reperfusion. This unique mechanism preserves protection even during diabetes where other protective strategies fail.

Keywords: near infrared light, nitrite reductase, ischemia and reperfusion injury, myoglobin, cardioprotection

\section{INTRODUCTION}

Restoration of blood flow to a region of previously ischemic myocardium (reperfusion) is a critical life-saving intervention against tissue necrosis, but reperfusion itself also results in significant damage to the myocardium. Many therapeutic strategies such as ischemic and volatile anesthetic pre- and postconditioning have been developed and are effective in healthy animal models but few have translated successfully to patients (Ludman et al., 2010). A major reason for the resistance to cardioprotection against infarction by physical or pharmacological stimuli is the advanced age and/or presence of comorbidities such as diabetes in patients. For example, endothelial dysfunction appears to contribute to the lack of protection by ischemic or anesthetic postconditioing in diabetes (Raphael et al., 2010; Przyklenk et al., 2011). Activation of endothelial nitric oxide synthase (eNOS) and pro-survival signaling pathways, together with alteration of mitochondrial bioenergetics, contribute to the mechanisms of various cardioprotective strategies against ischemia and reperfusion injury (Tsang et al., 2004; Mio et al., 2009; Ge et al., 2010). Although nitric oxide synthases (NOS) produce a large part of endogenous nitric oxide (NO), there is considerable interest in NOS-independent generation of $\mathrm{NO}$ in vivo, particularly during hypoxia or anoxia, where low oxygen tensions limit NOS activity (Godecke, 2006; Hendgen-Cotta et al., 2008, 2010). Interventions that can increase NO bioavailability have significant therapeutic potential. Under hypoxic conditions, heme-containing proteins such as myoglobin $(\mathrm{Mb})$ and hemoglobin $(\mathrm{Hb})$ exhibit nitrite reductase activity which results in an increase in NO bound to the heme iron of $\mathrm{Mb}$ and $\mathrm{Hb}$ (Gladwin et al., 2006; Hendgen-Cotta et al., 2008). We have recently found that far red/near infrared 
light (NIR) both in purified systems and in myocardium can release NO from nitrosyl hemes (Lohr et al., 2009). Further, NIR protected cardiomyocytes and the rabbit heart from hypoxia and reoxygenation injury in a $\mathrm{NO}$-dependent manner, reversible by NO scavenger CPTIO, and enhanced the protective effect of nitrite against ischemia and reperfusion injury of the rabbit heart (Lohr et al., 2009; Zhang et al., 2009).

NIR modulates biochemical systems by activating lightsensitive proteins harboring NIR-sensitive chromophores (Karu, 1999; Desmet et al., 2006). Previous studies suggested that NIR promotes cell survival during physiologic stress (Eells et al., 2003; Liang et al., 2008; Zhang et al., 2009). Repeated photostimulation of the myocardium has been demonstrated to be beneficial against long-term reperfusion injury in the rat and dog (Oron et al., 2001). For example, low-energy infrared (803 nm) laser irradiation delivered to the epicardium was shown to reduce scar formation and myocardial infarct size several weeks after prolonged coronary artery occlusion in dogs and rats (Oron et al., 2001). Aside from the heart, the beneficial effects of NIR light treatment have been studied in particular in a model of traumatic brain injury as well as in wound healing (Ankri et al., 2010; Naeser et al., 2011). NIR light treatment also improved the collateral blood vessel grow in a mouse model (tight skin mouse) of scleroderma (Zaidi et al., 2013). Frequently, the beneficial effects of NIR treatment have been associated with the stimulation of mitochondrial metabolism, particularly at the level of cytochrome c oxidase, complex IV of the electron transport chain (Karu, 2008). However, in a model of cardiac ischemia and reperfusion injury it is difficult to perceive how acceleration of cytochrome c oxidase at the time of reperfusion conveys protection to the heart. Rather, a mild reversible inhibition of the electron transport chain has been shown to reduce reactive oxygen species production during reperfusion, thereby increasing cardiomyocyte survival (Burwell et al., 2009). NO inhibits electron transport through competitive binding at complex IV and S-nitrosation at complex I (Piantadosi, 2012; Chouchani et al., 2013). Thus, we tested the hypothesis that brief exposure to NIR light at the time of reperfusion protects the heart in a wave length-dependent manner; and that this wave length dependence is paralleled by the release of NO from nitrosyl-heme proteins. We also examined whether NIR induced protection is maintained in a mouse model of acute hyperglycemia and diabetes $(\mathrm{db} / \mathrm{db})$ where protection by volatile anesthetics fail.

\section{MATERIALS AND METHODS}

All experimental procedures and protocols used in this investigation were reviewed and approved by the Animal Care and Use Committee of the Medical College of Wisconsin. Furthermore, all conformed to the Guiding Principles in the Care and Use of Animals of the American Physiologic Society and were in accordance with the Guide for the Care and Use of Laboratory Animals.

\section{MYOCARDIAL ISCHEMIA AND REPERFUSION INJURY IN MICE}

A murine model of myocardial ischemia and reperfusion injury was used as previously described (Ge et al., 2010). C57Bl/6 (wild type) mice, as well as $\mathrm{NOS}^{-/-}$and diabetic $\mathrm{db} / \mathrm{db}$ mice were used for these experiments. Glucose $(2 \mathrm{~g} / \mathrm{kg})$ was administered intraperitoneal $10 \mathrm{~min}$ before ischemia to produce hyperglycemia. Mice were anesthetized by intraperitoneal injection of sodium pentobarbital $(100 \mathrm{mg} / \mathrm{kg})$ and ventilated with room air supplemented with $100 \%$ oxygen at a rate of 100 breaths/min with a tidal volume of approximately $0.25 \mathrm{ml}$ using a rodent ventilator (Harvard Apparatus, South Natick, MA). Body temperature was maintained between $36.8^{\circ} \mathrm{C}$ and $37.5^{\circ} \mathrm{C}$. Myocardial ischemia was produced by occluding the left coronary artery (LAD) for $30 \mathrm{~min}$, and reperfusion was initiated by loosening the suture and continued for $3 \mathrm{~h}$.

\section{EXPERIMENTAL PROTOCOL}

Mice were randomly assigned to receive no irradiation (control) or NIR irradiation applied to the epicardial surface $\left(670 \mathrm{~nm}, 170 \mathrm{~mW} / \mathrm{cm}^{2}\right)$ with an LED array (NIR Products LLC, Milwaukee, WI) for $1 \mathrm{~min}$ before and through the first $4 \mathrm{~min}$ of reperfusion (energy-density equivalent to $51 \mathrm{~J} / \mathrm{cm}^{2}$ ). Separate experiments were performed to evaluate the energyand wavelength-dependence of NIR-mediated cardioprotection by varying the array $(670,740,830 \mathrm{~nm})$ and the output of the device.

$\mathrm{C} 57 \mathrm{BL} / 6, \mathrm{eNOS}^{-/-}$mice and $\mathrm{db} / \mathrm{db}$ mice were used to explore the dependence of NIR-mediated cardioprotection on eNOS and its efficacy in a diabetic animal.Acute hyperglycemia was induced by administration of D-glucose $(2 \mathrm{~g} / \mathrm{kg})$ in C57BL/6 mice $10 \mathrm{~min}$ before ischemia. Mannitol $(1.82 \mathrm{~g} / \mathrm{kg})$ was used for osmotic control in preliminary experiments in C57BL/6 mice both with and without NIR treatment and did not exhibit any significant effect compared to mice that did not receive mannitol. Pharmacological inhibition of eNOS was used to complement the experiments in eNOS $^{-/-}$mice and thus, C57BL/6 mice received $1 \mathrm{mg} / \mathrm{kg}$, i.v. of the non-selective NOS inhibitor L-NAME prior to LAD occlusion and reperfusion.

\section{DETERMINATION OF MYOCARDIAL INFARCT SIZE}

For infarct size measurements, the heart was first stained by cannulation of the aorta with a $1 \%$ solution of 2,3,5triphenytetrazolium chloride. Then the suture previously placed around the left descending coronary artery was retied and diluted phthalo blue dye was injected through the same cannula. As a result of these procedures, the non-ischemic portion of the left ventricle was stained dark blue. Viable myocardium within the area at risk was stained bright red, and infarcted tissue was light yellow. The heart was then excised and ventricles were cut into 4-5 uniform transverse slices of $2 \mathrm{~mm}$ thickness using a mouse heart matrix. Slices were then analyzed by planimetry.

\section{NITROSYL HEMOGLOBIN (HbNO) AND NITROSYL MYOGLOBIN (MbNO) PREPARATION}

Oxyhemoglobin purified from human blood according to a published procedure (Rossi-Fanelli et al., 1961) was deoxygenated, or solution of metmyoglobin (from horse skeletal muscle, Sigma) was reduced in an anaerobic chamber with $\mathrm{Na}_{2} \mathrm{~S}_{2} \mathrm{O}_{4}$ in phosphate buffered saline ( $\mathrm{PBS}, \mathrm{pH}$ 7.4). Then the heme proteins were nitrosylated by addition of equivalent concentration of highly concentrated PROLI NONOate (Cayman Chemicals, Ann 
Arbor, Mi) dissolved in $0.1 \mathrm{~N} \mathrm{NaOH}$. The process was spectrophotometrically followed. Solutions were made daily, and used immediately.

\section{NO-DEPENDENT CHEMILUMINESCENCE ANALYSIS}

A Sievers 280i Nitric Oxide Analyzer (General Electric, Boulder, $\mathrm{CO})$ was used to detect NO evolved from nitrosyl species as a consequence of NIR irradiation. $\mathrm{HbNO}$ or $\mathrm{MbNO}(3 \mathrm{ml}$ of $10 \mu \mathrm{M})$ was placed into the purge vessel of the analyzer, and externally irradiated at various powers and wavelengths for $1 \mathrm{~min}$. Detector response for NO liberated from known amounts of PROLI NONOate injected into PBS pH 7.4 was used as a basis of quantification.

\section{MEASUREMENT OF OXYGEN CONSUMPTION IN ISOLATED MITOCHONDRIA}

Rat heart mitochondria were isolated by differential centrifugation as previously reported (Pravdic et al., 2010). Mitochondrial oxygen consumption was measured with a Clark-type oxygen electrode (Hansatech Instruments, Norfolk, UK) at $30{ }^{\circ} \mathrm{C}$ in respiration buffer containing mitochondria at a final concentration of $1 \mathrm{mg}$ protein/mL. The mitochondrial respiration buffer was composed of $130 \mathrm{mM} \mathrm{KCl}, 5 \mathrm{mM} \mathrm{KH}_{2} \mathrm{PO}_{4}, 20 \mathrm{mM}$ MOPS, $2.5 \mathrm{mM}$ EGTA, $1 \mathrm{mM} \mathrm{Na}{ }_{4} \mathrm{P}_{2} \mathrm{O}_{7}$, and $0.1 \% \mathrm{BSA}$, at $\mathrm{pH}$ 7.4. State 2 respiration was initiated with $5 \mathrm{mM}$ pyruvate and $5 \mathrm{mM}$ malate as substrates. The adenosine diphosphate (ADP)-stimulated oxygen consumption (state 3 respiration) was measured in the presence of $250 \mu \mathrm{M}$ ADP. After hypoxia was reached mitochondria were incubated with deoxymyoglobin $(40 \mu \mathrm{M}$; prepared from myoglobin with sodium dithionite as reducing agent) and sodium nitrite $(20 \mu \mathrm{M})$ for $1 \mathrm{~min}$ and then exposed directly to NIR $\left(170 \mathrm{~mW} / \mathrm{cm}^{2}\right)$ for another min. After that the chamber was opened to allow reoxygenation. A faster rate of reoxygenation of the chamber indicated an inhibition of respiration.

\section{STATISTICAL ANALYSIS}

Statistical analysis of data within and between groups was performed with analysis of variance (ANOVA) for repeated measures followed by the Student-Newman-Keuls test. Changes were considered statistically significant when $P<0.05$. All data are expressed as mean \pm standard deviation (SD) unless otherwise indicated.

\section{RESULTS}

The mouse was chosen as model to determine the efficacy of NIR-mediated protection against cardiac ischemia and reperfusion injury in order to expand our findings on NIR-induced protection in rabbits and due to the advantage of the large availability of genetically engineered animals. Exposure to NIR for the last min of occlusion and first $4 \mathrm{~min}$ of reperfusion significantly $(P<0.05)$ reduced infarct size at the highest chosen irradiance $\left(170 \mathrm{~mW} / \mathrm{cm}^{2}\right.$, corresponding to $\left.51 \mathrm{~J} / \mathrm{cm}^{2}\right)$ compared to control experiments without NIR exposure $(31 \pm 7$ vs. $51 \pm 4 \%$ of left ventricular area of risk, Figure 1). The effect of NIR on infarct size was energy dependent. Myocardial infarct size was $59 \pm 5$ and 39 $\pm 6 \%$, at an irradiance of 10 and $27 \mathrm{~mW} / \mathrm{cm}^{2}$, corresponding to 3 and $8.1 \mathrm{~J} / \mathrm{cm}^{2}$ respectively. Thus, the threshold of cardioprotection appeared to occur at an irradiance level of $30 \mathrm{~mW} / \mathrm{cm}^{2}$.
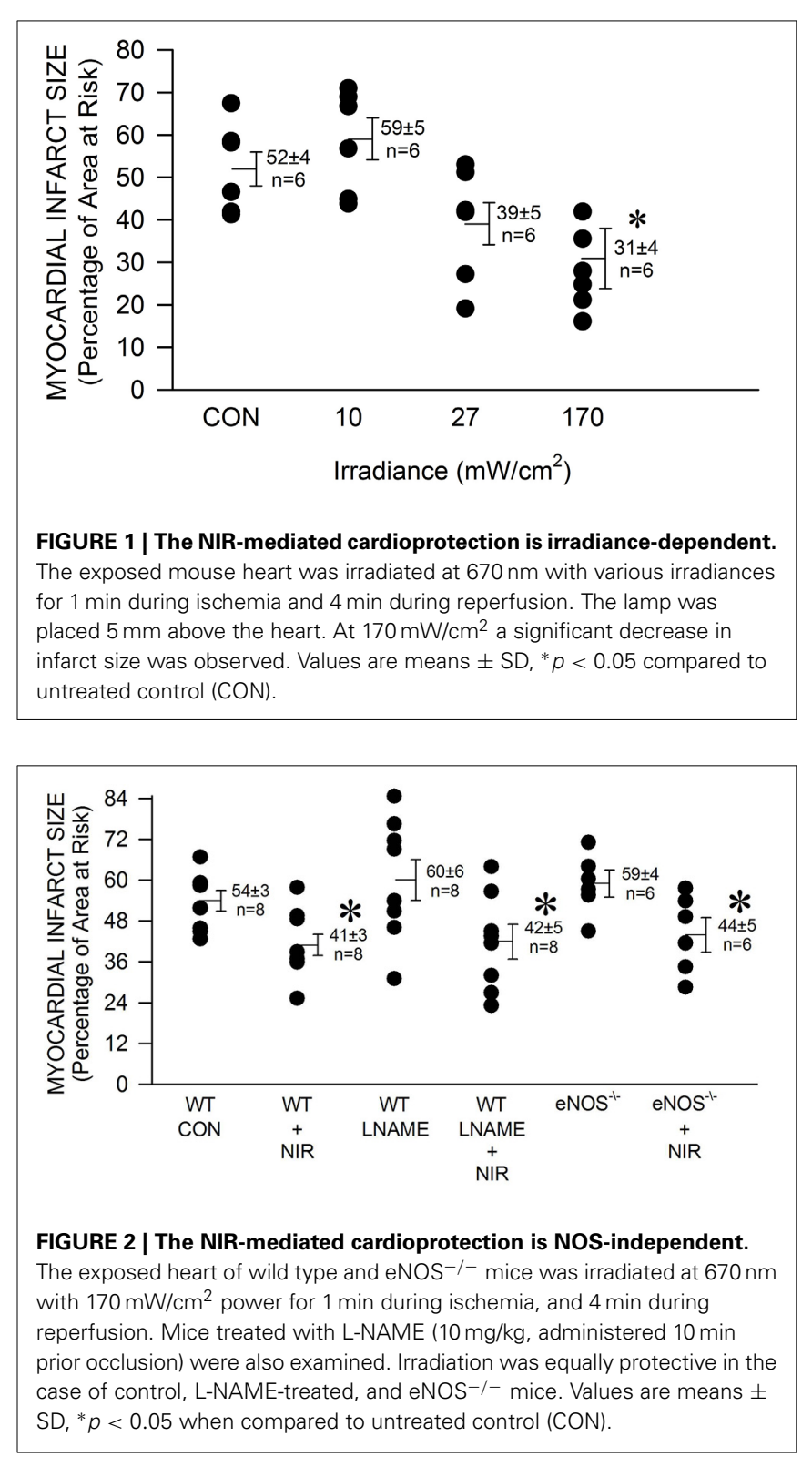

Importantly, no increase in epicardial surface temperature upon exposure to NIR was observed.

We then tested whether NOS is involved in the mechanism of NIR-induced protection. Pretreatment with the non-selective NOS inhibitor L-NAME had no effect alone $(60 \pm 6 \%$ infarct size of area at risk), nor did it inhibit NIR-mediated reduction in infarct size $(42 \pm 5 \%)$. Similarly, $\mathrm{eNOS}^{-/-}$mice were also protected against myocardial ischemia and reperfusion injury by NIR treatment ( $44 \pm 5 \%$ compared to $59 \pm 4 \%$ without treatment). An irradiance of $170 \mathrm{~mW} / \mathrm{cm}^{2}$ was applied in all cases. These data suggest that NIR-mediated cardioprotection is independent of the activity of NOS (Figure 2).

We recently reported (Lohr et al., 2009) that NIR light has the capacity to liberate NO from nitrosylated hemoglobin ( $\mathrm{HbNO}$ ) and myoglobin (MbNO). Here we examined the wavelength dependence of $\mathrm{NO}$ release and protection. A solution of 
HbNO or MbNO $(10 \mu \mathrm{M})$ was placed into the purge vessel of a chemiluminescence-based NO analyzer and subjected to irradiation for $1 \mathrm{~min}$. We observed 2-3 times more $\mathrm{NO}$ released at $670 \mathrm{~nm}$ compared to 740 and $830 \mathrm{~nm}$ at $10 \mathrm{~mW} / \mathrm{cm}^{2}$ irradiance (Figure 3A). As NIR does not have to penetrate tissue in these experiments, less irradiance compared to what is required for protection of the in vivo heart is needed. There was no significant difference between $\mathrm{NO}$ liberation from $\mathrm{HbNO}$ and MbNO. A similar trend was found with the wavelength dependence of NIR-induced reduction of infarct size. In contrast to $670 \mathrm{~nm}$ no significant protection was observed at 740 and $830 \mathrm{~nm}$ (Figure 3B).

Mitochondria are a potential therapeutic target of $\mathrm{NO}$ produced at the time of reoxygenation (Chouchani et al., 2013). Therefore, experiments were designed to establish the net outcome of NIR-enhanced nitrite reductase activity on mitochondrial respiration after hypoxia. We measured the reoxygenation rate of a mitochondrial suspension after hypoxia in the presence of deoxymyoglobin and nitrite, with and without NIR ( $660 \mathrm{~nm}, 50 \mathrm{~mW} / \mathrm{cm}^{2}$ ) (Figure 4). While $\mathrm{Mb}$ and nitrite induce inhibition of mitochondrial respiration alone (Shiva et al.,

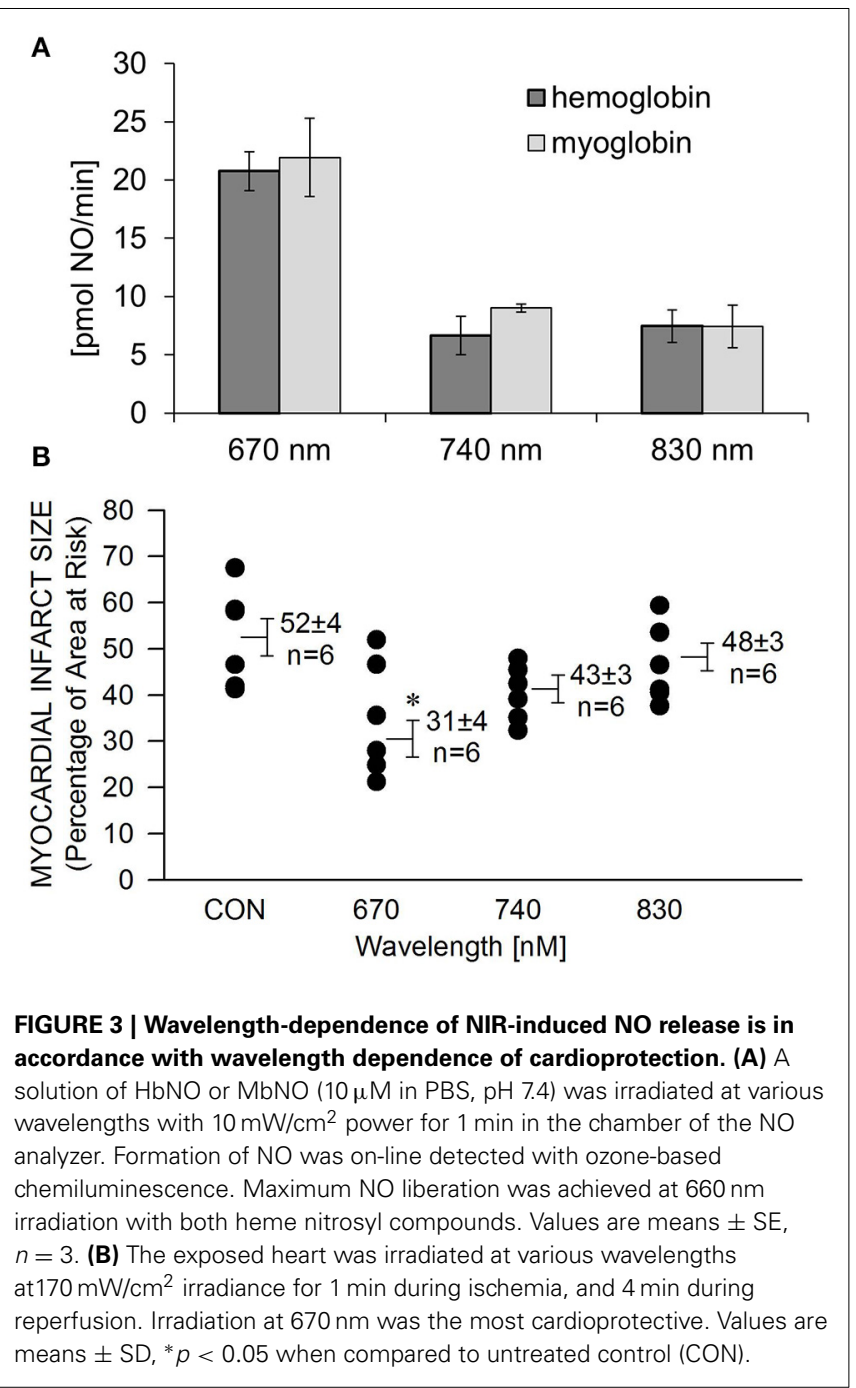

2007a,b; Hendgen-Cotta et al., 2008), we hypothesized that light enhances this inhibition through its action on $\mathrm{MbNO}$ formed as a consequence of nitrite reductase activity of heme. We found that NIR, while alone had no considerable effect, could potentiate the inhibition caused by $\mathrm{Mb}$ and nitrite at lower nitrite doses. It triggered a significantly faster reoxygenation and thereby a decrease in respiration rate in the presence of deoxymyoglobin and nitrite than solely deoxymyoglobin and nitrite would induce. A partial compensatory effect of $\mathrm{NO}$ bound to and released from complex IV cannot be excluded, however, in the investigated in vitro system $\mathrm{Mb}$ was present in wide excess over cyt $\mathrm{c}$ oxidase $(0.64 \mathrm{mg} / \mathrm{ml} \mathrm{Mb}$ vs. $1 \mathrm{mg} / \mathrm{ml}$ total mitochondrial protein), and NIR was switched off at the time of reoxygenation. Thus, NO released from Mb might partially bind to cytochrome c oxidase or mediate S-nitrosation of complex I at the beginning of reoxygenation, thereby accelerating reoxygenation and inhibiting respiration. The observed effect is relevant since a mild reversible inhibition of the mitochondrial electron transport chain during cardiac reperfusion has been shown to reduce reactive oxygen

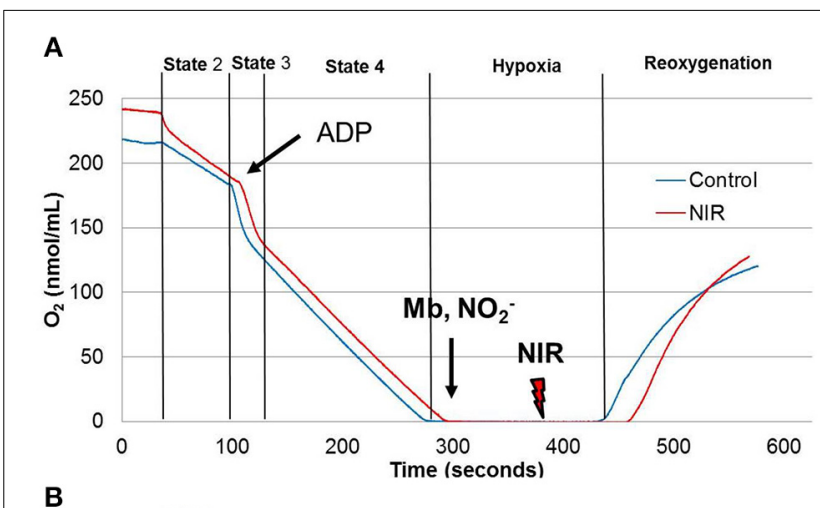

B

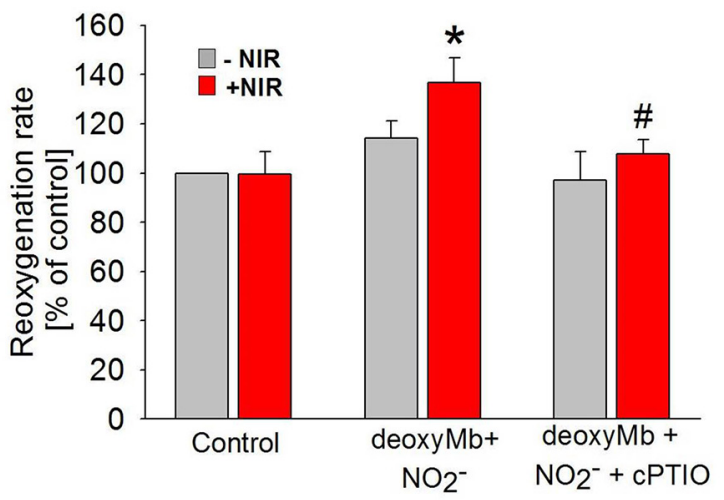

FIGURE 4 | NIR slows mitochondrial respiration in the presence of deoxymyoglobin and nitrite during reoxygenation. (A) Oxygen consumption of purified cardiac mitochondria was monitored with a Clarke-type oxygen electrode. At the beginning of ischemia, deoxyMb and sodium nitrite were added. During the second minute of ischemia before reoxygenation, mitochondria were exposed to NIR at $670 \mathrm{~nm}$ with $50 \mathrm{~mW} / \mathrm{cm}^{2}$ irradiance. (B) NIR caused an NO-dependent increase of reoxygenation rate (corresponding to a decrease of respiration) in the presence of deoxyMb and nitrite. The effect was reversed with NO scavenger CPTIO. Values are means $\pm \mathrm{SD}, n=6, * p<0.05$ when compared to control, ${ }^{\#} p<0.05$ when compared to NIR treated mitochondria in the absence of CPTIO. 
species production. In the presence of NO scavenger cPTIO $(10 \mu \mathrm{M})$, the NIR effect was reversed.

In diabetes, endothelial dysfunction, including defective NOS, is considered one of the causes for the failure of protective strategies such as ischemic or anesthetic pre- and postconditioning to reduce cardiac ischemia and reperfusion injury. Therefore, a NOS-independent mechanism of NO generation may allow NIR to reduce ischemia and reperfusion injury in the hyperglycemic or diabetic heart. Indeed, a similar degree of NIR-induced cardioprotection was observed in mice that were exposed to acute hyperglycemia ( $39 \pm 4 \%$ myocardial infarct size of area at risk vs. $52 \pm 2 \%$ without NIR) and in the diabetic $\mathrm{db} / \mathrm{db}$ mouse ( $43 \pm 4$ vs. $61 \pm 3 \%$ ) compared to the wild type mice ( $41 \pm 3 \%$ vs. $56 \pm 3 \%)$.

\section{DISCUSSION}

The current results demonstrate that a brief exposure to NIR immediately before and during early reperfusion protects the myocardium against infarction in an NOS-independent mechanism. Mitochondria are one potential therapeutic target of NIRinduced release of NO but other targets such as NO-sensitive guanylyl cyclase require further investigation. Importantly, NIR protects the hyperglycemic and diabetic heart. The absence of such protections has been one of the major hurdles in the implementation of pharmacological pre- and particularly postconditioning into the clinical setting.

In the nineteen nineties in Russia patients with coronary heart disease with prior myocardial infarction were exposed repeatedly to NIR by low-level laser therapy (LLLT) applied to the area of the heart on the skin. Lipid peroxidation was significantly reduced after NIR but little is known on whether cardiac function improved (Zubkova et al., 1993; Sorokina et al., 1997). In subsequent studies NIR was applied after chronic myocardial infarction in rat and dog models. NIR $\left(803 \mathrm{~nm}, 6 \mathrm{~mW} / \mathrm{cm}^{2}\right.$ at the surface of the myocardium for $3 \mathrm{~min}$, at 4-6 different locations) was applied twice, $15 \mathrm{~min}$ and 3 days after myocardial infarction, through the open chest directly onto the myocardium in dogs, and through the intercostal muscles in rats. Both mortality and infarct size were significantly reduced compare to untreated animals (Oron et al., 2001). Irradiation with NIR after myocardial infarction in rats resulted in a significant improved mitochondrial bioenergetics, and an increase in an inducible heat shock protein (HSP70), vascular endothelial growth factor (VEGF) and inducible nitric oxide synthase (iNOS) expression (Yaakobi et al., 2001). This was paralleled by a significant elevation in angiogenesis (Tuby et al., 2006). More recently, increased angiogenesis and collateralization upon NIR exposure $\left(670 \mathrm{~nm}, 50 \mathrm{~mW} / \mathrm{cm}^{2}\right.$, 10 min per day for 14 days) with LED have also been reported in the ischemic hind limb of mice and rabbits (Lohr et al., 2013). In a mouse model for systemic sclerosis, an autoimmune connective tissue disorder characterized by oxidative stress, impaired vascular function, and attenuated angiogenesis, NIR stimulated angiogenesis by increasing angiomotin and decreasing angiostatin expression in the ischemic hind limb (Zaidi et al., 2013).

In the present study we found that myocardial infarction can be prevented from occurring, or at least reduced by a one-time
NIR treatment right at the time of reoxygenation. It appears highly unlikely that NIR-induced reductions in infarct size were attributed to increased collateral perfusion as NIR-induced angiogenesis typically occurs as a result of sustained stimulation over several days. Therefore, the underlying mechanism of protection is likely different. Under hypoxic conditions heme-containing proteins such as myoglobin $(\mathrm{Mb})$ and hemoglobin $(\mathrm{Hb})$ exhibit nitrite reductase activity which results in an increase in NO liberation (Gladwin et al., 2006; Hendgen-Cotta et al., 2008). The NO formed may subsequently react with available deoxyHb or deoxyMb to yield iron-nitrosyl $\mathrm{Hb}(\mathrm{HbNO})$ or iron-nitrosyl $\mathrm{Mb}$ (MbNO). Thus, $\mathrm{HbNO}$ and $\mathrm{MbNO}$ may represent a significant storage pool of NO in the heart. Here we have demonstrated both for purified hemoglobin and myoglobin that NIR can decay nitrosyl heme and release NO in a wavelength-dependent manner. Importantly, the highest NO release was recorded at $670 \mathrm{~nm}$ where protection against ischemia and reperfusion injury was present (Figure 3). This further suggests a distinct mechanism from the previously reported protection through repeated NIR treatment in the permanently ligated heart where longer wavelengths were equally protective. We previously reported in the ischemic rabbit heart, after infusion of sodium nitrite, a large increase in nitrosyl heme formation as measured by electro paramagnetic resonance spectroscopy (EPR). The MbNO signal was reduced in the ischemic zone by NIR treatment suggesting dissociation of the heme-NO bond upon irradiation (Lohr et al., 2009).

Frequently, the beneficial effects of NIR treatment have been associated with the stimulation of mitochondrial metabolism, particularly at the level of cytochrome c oxidase, complex IV of the electron transport chain and concomitant enhancement of ATP synthesis (Karu, 2008). NIR may directly affect cytochrome c oxidase activity through one of its redox active metal centers. In addition, it has been suggested that NIR exerts its action on cytochrome c oxidase by a mechanism via NO release. The activated cytochrome $c$ oxidase may not only cause changes in electron transport chain activity, including ROS generation, but released NO is available for other biological processes such as vasodilation and gene expression. However, compared to potential NO release from $\mathrm{HbNO}$ or $\mathrm{MbNO}$ the relative amounts of NO in the case of cyt c oxidase is limited (Osipov et al., 2007). Further, it is difficult to perceive how acceleration of cytochrome c oxidase at the time of reperfusion conveys protection to the heart. Rather, a mild reversible inhibition of the electron transport chain has been shown to reduce reactive oxygen species production during reperfusion and increase cardiomyocyte survival (Burwell et al., 2009). This was confirmed in ischemic isolated mitochondria where, in the presence of deoxmyoglobin and sodium nitrite, a decrease in respiration was detected upon reoxygenation of mitochondria after application of NIR (Figure 4). NO signaling may lead to S-nitrosation of a cysteine residue in complex I that has been implicated in protection against cardiac ischemia and reperfusion injury (Cochain et al., 2013). Reversible S-nitrosation of complex I slows the reactivation of mitochondria during the crucial first minutes of the reperfusion of ischemic tissue, thereby decreasing ROS production, oxidative damage and tissue necrosis. 


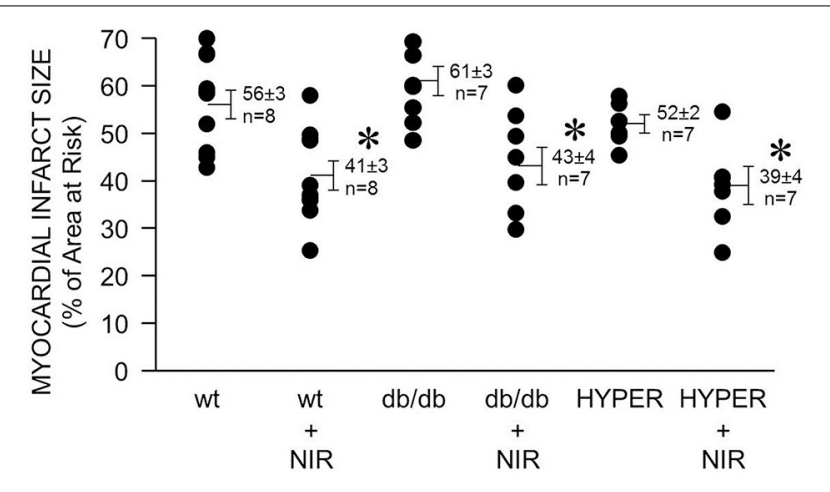

FIGURE 5 | NIR is cardioprotective in the presence of diabetes or hyperglycemia. The exposed heart of wild type, $\mathrm{db} / \mathrm{db}$, and hyperglycemic mice was irradiated at $670 \mathrm{~nm}$ with $170 \mathrm{~mW} / \mathrm{cm}^{2}$ irradiance for 1 min during ischemia, and 4 min during reperfusion. Light exposure resulted in a similarly effective decrease of infarct size in all three cases. Values are means $\pm S D, * p<0.05$ when compared to untreated wild type. to the heart with a flexible fiber optic NIR probe. The probe in the esophagus or stomach (when advanced) is immediately adjacent to the left atrium and the inferior and posterior walls of the left ventricle. Thus, the anterior wall that is frequently affected by myocardial infarction may be as much as $6 \mathrm{~cm}$ away from the probe. Still, it may not be necessary for NIR light to penetrate the area at risk directly. A remote effect of NIR, comparable to remote preconditioning, might still provide protection and lead to a reduction of infarct size. Signaling factors such as heat shock proteins or NO may mediate such effect.

\section{ACKNOWLEDGMENTS}

This work was supported in part by NIH (R01 HL098490), the Department of Veterans Affairs (CARA-026-10F) and the Roizen Anesthesia Research Foundation New Investigator Grant from the Society of Cardiovascular Anesthesiologists. The authors thank David A. Schwabe, John Tessmer, and John Krolikowski (Department of Anesthesiology, Medical College of Wisconsin, Milwaukee, Wisconsin) for technical assistance.

Due to the reasonably high tissue penetration paralleled by limited potential of tissue damage NIR is attractive for the use in ischemic heart disease. While the required light power needs to be verified for human cardiac use, by comparing animal studies through various species and experimental settings an irradiance of $10-100 \mathrm{~mW} / \mathrm{cm}^{2}$ for $2-10 \mathrm{~min}$ seems a reasonable starting point to achieve beneficial effects of NIR. A higher irradiance may be required for acute prevention of ischemia and reperfusion injury at the time of reperfusion. NIR treatment of the heart may be protective on patients after acute myocardial infarction or on ischemic heart conditions that are not accessible to current revascularization procedures. NIR could be particularly useful in the presence of comorbidities such as diabetes. Diabetes is an independent predictor of increased cardiovascular risk and myocardial infarct size is directly related to increases in blood glucose concentration in animals with or without diabetes (Van Der Horst et al., 2007). The mechanism of light-induced release of NO from iron-nitrosylated heme protein is likely to be maintained during diabetes and thus NIR may be protective from ischemia and reperfusion injury where other strategies such as ischemic and pharmacologic pre- and postconditioning fail (Kersten et al., 1998; Przyklenk et al., 2011). Indeed, we found that under acute hyperglycemia or in a mouse model of type 2 diabetes ( $\mathrm{db} / \mathrm{db}$ mouse) NIR exposure of the mouse heart at the time of reperfusion reduces infarct size significantly (Figure 5).

While there is substantial clinical promise for the use of NIR in heart disease several hurdles need to be considered and overcome. The technical challenge related to the application of NIR to the heart due to limited penetration through muscle and bone is an important issue to consider. Obvious scenarios would be application of light where needed during cardiac surgery such as coronary artery bypass that carry a significant increased risk of myocardial infarction, or heart transplantation. It might also be possible to apply NIR during a balloon angiography, using a catheter bearing fiber optic through which the light can be delivered to the infarcted area. In addition, authors' unpublished data on dogs demonstrate the feasibility of a transesophageal approach

\section{REFERENCES}

Ankri, R., Lubart, R., and Taitelbaum, H. (2010). Estimation of the optimal wavelengths for laser-induced wound healing. Lasers Surg. Med. 42, 760-764. doi: 10.1002/lsm.20955

Burwell, L. S., Nadtochiy, S. M., and Brookes, P. S. (2009). Cardioprotection by metabolic shut-down and gradual wake-up. J. Mol. Cell. Cardiol. 46, 804-810. doi: 10.1016/j.yjmcc.2009.02.026

Chouchani, E. T., Methner, C., Nadtochiy, S. M., Logan, A., Pell, V. R., Ding, S., et al. (2013). Cardioprotection by S-nitrosation of a cysteine switch on mitochondrial complex I. Nat. Med. 19, 753-759. doi: 10.1038/nm.3212

Cochain, C., Channon, K. M., and Silvestre, J. S. (2013). Angiogenesis in the infarcted myocardium. Antioxid. Redox Signal. 18, 1100-1113. doi: 10.1089/ars.2012.4849

Desmet, K. D., Paz, D. A., Corry, J. J., Eells, J. T., Wong-Riley, M. T., Henry, M. M., et al. (2006). Clinical and experimental applications of NIR-LED photobiomodulation. Photomed. Laser Surg. 24, 121-128. doi: 10.1089/pho.2006. 24.121

Eells, J. T., Henry, M. M., Summerfelt, P., Wong-Riley, M. T., Buchmann, E. V., Kane, M., et al. (2003). Therapeutic photobiomodulation for methanolinduced retinal toxicity. Proc. Natl. Acad. Sci. U.S.A. 100, 3439-3444. doi: 10.1073/pnas.0534746100

Ge, Z. D., Pravdic, D., Bienengraeber, M., Pratt, P. F. Jr., Auchampach, J. A., Gross, G. J., et al. (2010). Isoflurane postconditioning protects against reperfusion injury by preventing mitochondrial permeability transition by an endothelial nitric oxide synthase-dependent mechanism. Anesthesiology 112, 73-85. doi: 10.1097/ALN.0b013e3181c4a607

Gladwin, M. T., Raat, N. J., Shiva, S., Dezfulian, C., Hogg, N., Kim-Shapiro, D. B., et al. (2006). Nitrite as a vascular endocrine nitric oxide reservoir that contributes to hypoxic signaling, cytoprotection, and vasodilation. Am. J. Physiol. Heart Circ. Physiol. 291, H2026-H2035. doi: 10.1152/ajpheart. 00407.2006

Godecke, A. (2006). On the impact of NO-globin interactions in the cardiovascular system. Cardiovasc. Res. 69, 309-317. doi: 10.1016/j.cardiores.2005. 10.010

Hendgen-Cotta, U. B., Kelm, M., and Rassaf, T. (2010). A highlight of myoglobin diversity: the nitrite reductase activity during myocardial ischemia-reperfusion. Nitric Oxide 22, 75-82. doi: 10.1016/j.niox.2009.10.003

Hendgen-Cotta, U. B., Merx, M. W., Shiva, S., Schmitz, J., Becher, S., Klare, J. P., et al. (2008). Nitrite reductase activity of myoglobin regulates respiration and cellular viability in myocardial ischemia-reperfusion injury. Proc. Natl. Acad. Sci. U.S.A. 105, 10256-10261. doi: 10.1073/pnas.0801336105

Karu, T. (1999). Primary and secondary mechanisms of action of visible to nearIR radiation on cells. J. Photochem. Photobiol. B 49, 1-17. doi: 10.1016/S10111344(98)00219-X 
Karu, T. I. (2008). Mitochondrial signaling in mammalian cells activated by red and near-IR radiation. Photochem. Photobiol. 84, 1091-1099. doi: 10.1111/j.17511097.2008.00394.x

Kersten, J. R., Schmeling, T. J., Orth, K. G., Pagel, P. S., and Warltier, D. C. (1998). Acute hyperglycemia abolishes ischemic preconditioning in vivo. Am. J. Physiol. 275, H721-H725.

Liang, H. L., Whelan, H. T., Eells, J. T., and Wong-Riley, M. T. (2008). Near-infrared light via light-emitting diode treatment is therapeutic against rotenone- and 1-methyl-4-phenylpyridinium ion-induced neurotoxicity. Neuroscience 153, 963-974. doi: 10.1016/j.neuroscience.2008.03.042

Lohr, N. L., Keszler, A., Pratt, P., Bienengraber, M., Warltier, D. C., and Hogg, N. (2009). Enhancement of nitric oxide release from nitrosyl hemoglobin and nitrosyl myoglobin by red/near infrared radiation: potential role in cardioprotection. J. Mol. Cell. Cardiol. 47, 256-263. doi: 10.1016/j.yjmcc.2009. 03.009

Lohr, N. L., Ninomiya, J. T., Warltier, D. C., and Weihrauch, D. (2013). Far red/near infrared light treatment promotes femoral artery collateralization in the ischemic hindlimb. J. Mol. Cell. Cardiol. 62, 36-42. doi: 10.1016/j.yjmcc.2013.05.007

Ludman, A. J., Yellon, D. M., and Hausenloy, D. J. (2010). Cardiac preconditioning for ischaemia: lost in translation. Dis. Model. Mech. 3, 35-38. doi: 10.1242/dmm.003855

Mio, Y., Shim, Y. H., Richards, E., Bosnjak, Z. J., Pagel, P. S., and Bienengraeber, M. (2009). Xenon preconditioning: the role of prosurvival signaling, mitochondrial permeability transition and bioenergetics in rats. Anesth. Analg. 108, 858-866. doi: 10.1213/ane.0b013e318192a520

Naeser, M. A., Saltmarche, A., Krengel, M. H., Hamblin, M. R., and Knight, J. A. (2011). Improved cognitive function after transcranial, light-emitting diode treatments in chronic, traumatic brain injury: two case reports. Photomed. Laser Surg. 29, 351-358. doi: 10.1089/pho.2010.2814

Oron, U., Yaakobi, T., Oron, A., Hayam, G., Gepstein, L., Rubin, O., et al. (2001). Attenuation of infarct size in rats and dogs after myocardial infarction by low-energy laser irradiation. Lasers Surg. Med. 28, 204-211. doi: 10.1002/ lsm.1039

Osipov, A. N., Borisenko, G. G., and Vladimirov, Y. A. (2007). Biological activity of hemoprotein nitrosyl complexes. Biochemistry (Mosc.) 72, 1491-1504. doi: 10.1134/S0006297907130068

Piantadosi, C. A. (2012). Regulation of mitochondrial processes by protein S-nitrosylation. Biochim. Biophys. Acta 1820, 712-721. doi: 10.1016/j.bbagen.2011.03.008

Pravdic, D., Mio, Y., Sedlic, F., Pratt, P. F., Warltier, D. C., Bosnjak, Z. J., et al. (2010). Isoflurane protects cardiomyocytes and mitochondria by immediate and cytosol-independent action at reperfusion. Br. J. Pharmacol. 160, 220-232. doi: 10.1111/j.1476-5381.2010.00698.x

Przyklenk, K., Maynard, M., Greiner, D. L., and Whittaker, P. (2011). Cardioprotection with postconditioning: loss of efficacy in murine models of type-2 and type-1 diabetes. Antioxid. Redox Signal. 14, 781-790. doi: 10.1089/ars.2010.3343

Raphael, J., Gozal, Y., Navot, N., and Zuo, Z. (2010). Hyperglycemia inhibits anesthetic-induced postconditioning in the rabbit heart via modulation of phosphatidylinositol-3-kinase/Akt and endothelial nitric oxide synthase signaling. J. Cardiovasc. Pharmacol. 55, 348-357. doi: 10.1097/FJC.0b013e $3181 \mathrm{~d} 26583$

Rossi-Fanelli, A., Antonini, E., and Caputo, A. (1961). Studies on the relations between molecular and functional properties of hemoglobin. I. The effect of salts on the molecular weight of human hemoglobin. J. Biol. Chem. 236, 391-396.
Shiva, S., Huang, Z., Grubina, R., Sun, J., Ringwood, L. A., MacArthur, P. H., et al. (2007b). Deoxymyoglobin is a nitrite reductase that generates nitric oxide and regulates mitochondrial respiration. Circ. Res. 100, 654-661. doi: 10.1161/01.RES.0000260171.52224.6

Shiva, S., Sack, M. N., Greer, J. J., Duranski, M., Ringwood, L. A., Burwell, L., et al. (2007a). Nitrite augments tolerance to ischemia/reperfusion injury via the modulation of mitochondrial electron transfer. J. Exp. Med. 204, 2089-2102. doi: 10.1084/jem.20070198

Sorokina, E. I., Kenevich, N. A., Zubkova, S. M., and Minenkov, A. A. (1997). [The comparative action of laser radiation in different spectra on patients with ischemic heart disease]. Vopr. Kurortol. Fizioter. Lech. Fiz. Kult. 11-13.

Tsang, A., Hausenloy, D. J., Mocanu, M. M., and Yellon, D. M. (2004). Postconditioning: a form of "modified reperfusion" protects the myocardium by activating the phosphatidylinositol 3-kinase-Akt pathway. Circ. Res. 95, 230-232. doi: 10.1161/01.RES.0000138303.76488.fe

Tuby, H., Maltz, L., and Oron, U. (2006). Modulations of VEGF and iNOS in the rat heart by low level laser therapy are associated with cardioprotection and enhanced angiogenesis. Lasers Surg. Med. 38, 682-688. doi: 10.1002/lsm. 20377

Van Der Horst, I. C., Nijsten, M. W., Vogelzang, M., and Zijlstra, F. (2007). Persistent hyperglycemia is an independent predictor of outcome in acute myocardial infarction. Cardiovasc. Diabetol. 6:2. doi: 10.1186/14752840-6-2

Yaakobi, T., Shoshany, Y., Levkovitz, S., Rubin, O., Ben Haim, S. A., and Oron, U. (2001). Long-term effect of low energy laser irradiation on infarction and reperfusion injury in the rat heart. J. Appl. Physiol. 90, 2411-2419.

Zaidi, M., Krolikowki, J. G., Jones, D. W., Pritchard, K. A. Jr., Struve, J., Nandedkar, S. D., et al. (2013). Transient repetitive exposure to low level light therapy enhances collateral blood vessel growth in the ischemic hindlimb of the tight skin mouse. Photochem. Photobiol. 89, 709-713. doi: 10.1111/ php.12024

Zhang, R., Mio, Y., Pratt, P. F., Lohr, N., Warltier, D. C., Whelan, H. T., et al. (2009). Near infrared light protects cardiomyocytes from hypoxia and reoxygenation injury by a nitric oxide dependent mechanism. J. Mol. Cell. Cardiol. 46, 4-14. doi: 10.1016/j.yjmcc.2008.09.707

Zubkova, S. M., Sorokina, E. I., Kenevich, N. A., Tupitsyna, I., and Minenkov, A. A. (1993). [The blood antioxidant system in patients with ischemic heart disease undergoing laser therapy]. Vopr. Kurortol. Fizioter. Lech. Fiz. Kult. 4-7.

Conflict of Interest Statement: The authors declare that the research was conducted in the absence of any commercial or financial relationships that could be construed as a potential conflict of interest.

Received: 31 May 2014; accepted: 26 July 2014; published online: 22 August 2014. Citation: Keszler A, Brandal G, Baumgardt S, Ge Z-D, Pratt PF, Riess ML and Bienengraeber $M$ (2014) Far red/near infrared light-induced protection against cardiac ischemia and reperfusion injury remains intact under diabetic conditions and is independent of nitric oxide synthase. Front. Physiol. 5:305. doi: 10.3389/fphys. 2014.00305

This article was submitted to Mitochondrial Research, a section of the journal Frontiers in Physiology.

Copyright (๑) 2014 Keszler, Brandal, Baumgardt, Ge, Pratt, Riess and Bienengraeber. This is an open-access article distributed under the terms of the Creative Commons Attribution License (CC BY). The use, distribution or reproduction in other forums is permitted, provided the original author(s) or licensor are credited and that the original publication in this journal is cited, in accordance with accepted academic practice. No use, distribution or reproduction is permitted which does not comply with these terms. 